03

\title{
K вопросу о вычислении времени остановки вращающегося в вязком континууме цилиндрического тела и времени увлечения соосного с ним внешнего цилиндра
}

\author{
(C) C.O. Гладков
}

Московский авиационный институт (национальный исследовательский университет) (МАИ), 125997 Москва, Россия

e-mail: sglad@newmail.ru

(Поступило в Редакцию 24 мая 2017 г.)

Найдено распределение скоростей вблизи поверхности аксиально симметричного тела, вращающегося в вязкой среде с частотой $\omega$, направленной вдоль его оси. Вычислена диссипативная функция, и с ее помощью найдено уравнение движения, из которого получено аналитическое выражение для времени торможения тела до его полной остановки. Из решения нестационарного уравнения Навье-Стокса вычислено время увлечения внешнего соосного с ним неподвижного цилиндра.

DOI: $10.21883 /$ JTF.2018.03.45587.2349

\section{Введение}

Задача, о которой пойдет сейчас речь, не новая. Например, в классической монографии [1] в §18 приведено решение задачи о распределении поля скоростей между двумя вращающимися с разными угловыми скоростями коаксиальными цилиндрами радиусов $R_{1}$ и $R_{2}$. При стремлении радиуса внешнего цилиндра $R_{2}$ к бесконечности мы придем к нашей задаче. Однако наш интерес к ней появился не совсем случайно и был обоснован следующими соображениями. Во-первых, вызывает любопытство решение этой задачи другим способом, а именно вычисление распределения скоростей с помощью предположения, что его можно найти исходя из формулы $\mathbf{v}=\operatorname{rotrot}(f(r)[\boldsymbol{\omega} \times \mathbf{r}])$ где $\boldsymbol{\omega}$ - частота вращения, а $f(r)$ - неизвестная функция, зависящая от расстояния $r$, отсчитываемого от оси вращения. А вовторых, очень интересен вывод уравнения движения вращающегося осесимметричного тела в вязком континууме и оценка его времени остановки. Эта задача будет решена в первой части работы. Во второй части работы мы рассмотрим более сложную задачу и оценим время, по прошествии которого начнется процесс вовлечения во вращение неподвижного внешнего цилиндра с радиусом $R_{2}$, соосного с вращающимся внутренним цилиндром.

\section{1. Вычисление времени остановки вращающегося в вязком континууме цилиндрического тела}

Для решения этой задачи нам потребуется вывод динамического уравнения движения с учетом сил вязкого сопротивления, обусловливающими возникший при этом тормозящий момент. Прежде, чем приступить к непосредственным вычислениям, хотелось бы сразу подчеркнуть, что нагревом жидкости, возникающим при вращении цилиндра, мы будем пренебрегать.

В отличие от [1] тормозящий момент будет вычислен не с помощью тензора вязких напряжений, а с помощью диссипативной функции $\dot{Q}$, согласно которой его можно найти как производную по частоте, а именно

$$
\mathbf{M}_{c}=\frac{\partial \dot{Q}}{\partial \omega} .
$$

В случае несжимаемой жидкости диссипативная функция в криволинейных координатах имеет вид

$$
\dot{Q}=\frac{\eta}{2} \int_{V}\left(v_{i, k}+v_{k, i}\right)^{2} d V,
$$

где $\eta$ - динамическая вязкость, $V$ - весь объем вне тела вращения, а $v_{i, k}$ - ковариантные производные, определяемые соотношением

$$
v_{i, k}=\frac{\partial v_{i}}{\partial x^{k}}-\Gamma_{i k l} v^{l}
$$

где $\Gamma_{i k l}$ - символы Кристоффеля первого рода [2].

Для решения задачи запишем уравнение НавьеСтокса для несжимаемой жидкости в общем виде в криволинейной системе координат как

$$
\begin{aligned}
\rho \frac{\partial v_{i}}{\partial t} & +\rho v^{k}\left(\frac{\partial v_{i}}{\partial x^{k}}-\Gamma_{i k l} v^{l}\right)= \\
& -\frac{\partial P}{\partial x^{i}}+\rho g_{i}+\eta\left[\Delta v_{i}-v^{s} \frac{\partial \Gamma_{i k s}}{\partial x^{k}}\right. \\
& \left.-\Gamma_{i k s} \frac{\partial v^{s}}{\partial x^{k}}+g^{n p} \Gamma_{i k n} \frac{\partial v_{p}}{\partial x^{k}}-\Gamma_{i k n} \Gamma_{l s}^{n} v^{s}\right],
\end{aligned}
$$

где $\Gamma_{k l}^{i}$ - символы Кристоффеля второго рода [2], $\rho$ плотность континуума, $P$ - давление, $\mathbf{g}-$ ускорение силы тяжести, $g^{i k}$ - контравариантный метрический 
тензор. В нашей задаче уравнение (4) для стационарного решения в случае малых чисел Рейнольдса с учетом центростремительной силы, действующей на единицу объема жидкости, увлекаемой вращением тела, можно записать в значительно более простом и привычном виде, учитывая, что $\mathbf{g} \perp \mathbf{r}$, а именно

$$
\nabla P=\eta \Delta \mathbf{v}-\rho \frac{v^{2}}{r^{2}} \mathbf{r}
$$

где скорость $v=\sqrt{v_{\varphi}^{2}+v_{r}^{2}}$. Ее радиальная составляющая $v_{r}=\frac{d r}{d t}=0$, а тангенциальная $v_{\varphi}-$ будет найдена из решения уравнения (5), и которая описывается чисто радиальным распределением скоростей (см. ниже). Поэтому $v=v_{\varphi}(r)$ для всех $r \geq R$. Взяв операцию rot от обеих сторон уравнения (5) и в силу того, что ротор от последнего слагаемого в (5) тождественно равен нулю, находим

$$
\Delta \operatorname{rotv}=0 .
$$

С целью решения уравнения (6) предположим, что скорость можно найти как

$$
\mathbf{v}=\operatorname{rotrot}(f(r)[\omega \times \mathbf{r}])
$$

(см. также работу [3]). Поскольку

$$
\operatorname{rotrot} \mathbf{A}=\operatorname{graddiv} \mathbf{A}-\Delta \mathbf{A},
$$

то при подстановке (7) в (6) градиент исчезает и мы получаем, что

$$
\Delta^{2} \operatorname{rot}(f(r)[\omega \times \mathbf{r}])=0 .
$$

Так как

$$
\operatorname{rot}(f(r)[\boldsymbol{\omega} \times \mathbf{r}])=(\boldsymbol{\omega} \cdot \mathbf{r}) \mathbf{r} \frac{f^{\prime}}{r}-\boldsymbol{\omega}\left(2 f+r f^{\prime}\right),
$$

a $\omega \perp \mathbf{r}$, то

$$
\operatorname{rot}(f(r)[\omega \times \mathbf{r}])=-\omega\left(2 f+r f^{\prime}\right) .
$$

Поэтому с учетом того, что $\boldsymbol{\omega}=$ const и ее можно вынести за знак оператора Лапласа, приходим к бигармоническому уравнению

$$
\Delta^{2}\left(2 f+r f^{\prime}\right)=0 .
$$

В цилиндрической системе координат для радиальной части оператора Лапласа имеем

$$
\Delta=\frac{1}{r} \frac{d}{d r} r \frac{d}{d r}
$$

Поэтому уравнение (9) сильно упрощается и будет таким

$$
\left(\frac{1}{r} \frac{d}{d r} r \frac{d}{d r}\right)\left(\frac{1}{r} \frac{d}{d r} r \frac{d}{d r}\right)\left(2 f+r f^{\prime}\right)=0 .
$$

После четырехкратного интегрирования элементарно получаем

$$
r f^{\prime}+2 f=C_{1} r^{2} \ln r+C_{2} r^{2}+C_{3} \ln r+C_{4},
$$

где $C_{1,2,3,4}$ - константы интегрирования. Решая теперь неоднородное уравнение первого порядка (10), приходим к такому результату

$$
f(r)=a r^{2} \ln r+b r^{2}+c \ln r+d+\frac{e}{r^{2}},
$$

где $a, b, c, d, e-$ новые константы интегрирования. В силу того, что на бесконечности скорость должна обращаться в нуль, из решения (11) сразу же следует вывод о том, что $a=b=0$. Далее. Как видно из (7),

$$
\mathbf{v}=[\omega \times \mathbf{r}] \frac{\left(3 f^{\prime}+r f^{\prime \prime}\right)}{r},
$$

a потому можно считать, что $d=0$. Согласно (12), скорость имеет единственную отличную от нуля компоненту $v_{\varphi}$, т.е.

$$
\left\{\begin{array}{l}
v_{r}=0 \\
v_{\varphi}=\omega\left(3 f^{\prime}+r f^{\prime \prime}\right), \\
v_{z}=0
\end{array}\right.
$$

С учетом сказанного, подставляя сюда решение (11) в виде $f(r)=c \ln r+\frac{e}{r^{2}}$, находим

$$
v_{\varphi}=\frac{2 c \omega}{r} .
$$

Так как константа $e$ сюда не входит, мы имеем право положить ее равной нулю. То есть для функции $f(r)=a r^{2} \ln r+b r^{2}+c \ln r+d+\frac{e}{r^{2}}$ получим окончательное решение в виде

$$
f(r)=c \ln r .
$$

Из граничного условия $\left.v_{\varphi}\right|_{r=R}=\omega R$ с помощью (14) будем иметь, что $c=\frac{R^{2}}{2}$. Поэтому искомое решение есть $v_{\varphi}=\frac{R^{2} \omega}{r}$. Следовательно, окончательно из (13) находим

$$
\left\{\begin{array}{l}
v_{r}=v_{z}=0, \\
v_{\varphi}=\frac{R^{2} \omega}{r} .
\end{array}\right.
$$

Распределение скоростей в виде (16) дает ответ на вопрос о функциональной зависимости скорости жидкости вблизи поверхности вращающегося цилиндра. Заметим, что решение (16) описывает распределение не только для цилиндрического тела, но и для любого тела вращения, для которого радиус $R$ является функцией от $r$, т.е. $R=R(r)$. Поэтому решение (16) в этом общем случае можно записать в более общем виде

$$
\left\{\begin{array}{l}
v_{r}=v_{z}=0, \\
v_{\varphi}=\frac{\omega R^{2}(r)}{r} .
\end{array}\right.
$$


Что касается распределения давления около поверхности цилиндра, то его также можно легко найти с помощью уравнения (5). Действительно, взяв от его обеих частей операцию div, получаем с учетом решения (16)

$$
\Delta P=\rho \operatorname{div}\left(\frac{v_{\varphi}^{2}}{r^{2}} \mathbf{r}\right)=\rho \omega^{2} R^{4} \operatorname{div}\left(\frac{\mathbf{r}}{r^{4}}\right)=-2 \frac{\rho \omega^{2} R^{4}}{r^{4}}
$$

Решение уравнения (18) можно элементарно найти, записав оператор Лапласа в цилиндрической системе координат и оставив только радиальную часть, т.е.

$$
\frac{1}{r} \frac{d}{d r} r \frac{d P}{d r}=-\frac{2 \rho \omega^{2} R^{4}}{r^{4}} .
$$

Интегрируя по $r$, найдем в результате

$$
P(r)=P_{0}-\frac{\rho \omega^{2} R^{4}}{2 r^{2}} .
$$

Чтобы получить теперь диссипативную функцию, согласно (2), в определение которой входят символы Кристоффеля, согласно (3), то нам надо вычислить все отличные от нуля компоненты $\Gamma_{i k l}$. Согласно их определению,

$$
\Gamma_{i k l}=\frac{\partial x_{n}}{\partial x^{i}} \frac{\partial^{2} x_{n}}{\partial x^{k} \partial x^{l}} .
$$

А потому для преобразований $x=r \cos \varphi, y=r \sin \varphi$ легко получить следующие ненулевые компоненты символа Кристоффеля:

$$
\begin{gathered}
\Gamma_{\varphi \varphi}^{r}=\Gamma_{r \varphi \varphi}=-\frac{1}{r}, \\
\Gamma_{\varphi r}^{\varphi}=\Gamma_{\varphi r}^{\varphi}=\Gamma_{\varphi \varphi r}=\Gamma_{\varphi r \varphi}=\frac{1}{r} .
\end{gathered}
$$

В результате простых манипуляций находим следующее выражение для диссипативной функции:

$$
\begin{aligned}
\dot{Q} & =\eta \int_{V}\left[\left(\frac{\partial v_{\varphi}}{\partial r}\right)^{2}+v_{\varphi}^{2}\left(\Gamma_{\varphi r \varphi}+\Gamma_{r \varphi \varphi}\right)^{2}\right] d V \\
& =\eta \int_{V}\left(\frac{\partial v_{\varphi}}{\partial r}\right)^{2} d V .
\end{aligned}
$$

Переходя здесь к цилиндрической системе координат, будем иметь

$$
\dot{Q}=\eta \omega^{2} R^{4} \int_{V} \frac{d V}{r^{4}}=2 \pi \eta l \omega^{2} R^{4} \int_{R}^{\infty} \frac{d r}{r^{3}}=\pi \eta l \omega^{2} R^{2} .
$$

Чтобы получить теперь уравнение движения, запишем кинетическую энергию вращающегося цилиндра как

$$
E_{k}=\frac{J \omega^{2}}{2}
$$

где его момент инерции определяется в виде

$$
J=\frac{\pi}{2} \rho l R^{4}
$$

Чтобы теперь получить интересующее нас уравнение движения, представим его сначала в общем неявном виде, как сумму

$$
\dot{E}_{k}+\dot{Q}=0
$$

Подставляя сюда (22) и (23), находим в результате

$$
\dot{\omega}+\frac{\omega}{\tau}=0,
$$

где время остановки $\tau$ с учетом явного выражения для момента инерции будет определяться следующим образом:

$$
\tau=\frac{\rho R^{2}}{4 \eta}
$$

Если воспользоваться, например, значениями параметров $\rho=7.8 \mathrm{~g} / \mathrm{cm}^{3}, R=10 \mathrm{~cm}, \eta=1 \mathrm{~g} / \mathrm{cm} \cdot \mathrm{c}$, из (25) получим оценку $\tau \approx 3 \mathrm{~min}$.

Если представить себе движущийся поступательно цилиндр, одновременно вращающийся вокруг своей собственной оси вдоль направления движения, то подобная задача, как это становится вполне очевидным, будет иметь уже чисто практическое значение (к примеру летящий снаряд). Для ее решения удобно перейти в систему отсчета, связанную с чисто поступательным движением цилиндра, а потому для оценки времени релаксации (поскольку оно не зависит от выбора системы отсчета и инвариантно в любой системе координат) мы имеем право воспользоваться формулой (25).

\section{2. Оценка времени вовлечения во вращение неподвижного внешнего цилиндра}

Рассмотрим теперь другую задачу. Представим себе два коаксиальных и вертикально расположенных цилиндра, внутренний и внешний радиусы которых есть соответственно $R_{1}$ и $R_{2}$. Пространство между ними заполнено жидкостью с динамической вязкостью $\eta$. Внешний цилиндр покоится, а внутренний вращается с постоянной угловой скоростью $\omega$ вдоль направления оси цилиндра, которую будем обозначать буквой $z$. Поставим перед собой задачу выяснить, через какое время после начала вращения внутреннего цилиндра начнет вовлекаться в движение внешний неподвижный цилиндр. Надо сказать, что сформулированная задача не является единственной в своем роде, а объединяет некоторый класс подобных проблем, в частности, весьма известную задачу о синхронизации маятников механических часов, подвешенных на одной балке. Заметим, однако, что физика в этом случае несколько отличается от рассматриваемого нами примера, хотя ход ее решения и смысл задачи вполне понятны.

Надо сказать, что в отличие от задачи, рассмотренной в первой части работы, эта задача имеет принципиально нестационарный характер и требует решения уравнения Навье-Стокса с учетом его левой части, т.е. с учетом 
субстанциональной производной. В общем случае для несжимаемой жидкости уравнение можно представить в виде

$$
\frac{\partial \mathbf{v}}{\partial t}+(\mathbf{v} \cdot \nabla) \mathbf{v}=-\frac{\nabla P}{\rho}+\mathbf{g}+v \Delta \mathbf{v},
$$

где $\mathbf{g}$ - ускорение силы тяжести, и помнить про уравнение $\operatorname{divv}=0$.

В силу осевой симметрии задачи вектор скорости жидкости, вовлекаемой в движение вращающимся цилиндром, в цилиндрической системе координат должен иметь такой вид

$$
\mathbf{v}=\left(0, v_{\varphi}(r, t), v_{z}(r, t)\right),
$$

обеспечивающий автоматическое выполнение условия несжимаемости жидкости $\operatorname{divv}=0$, а компоненты скорости $v_{\varphi}(r, t)$ и $v_{z}(r, t)$ следует найти.

Для решения задачи воспользуемся общим уравнением (4), справедливым в произвольной криволинейной системе координат. С учетом найденных выше символов Кристоффеля для проекций уравнения (4) на оси $\varphi, z$ и $r$ имеем

$$
\left\{\begin{array}{l}
\frac{\partial v_{\varphi}}{\partial t}=v\left(\Delta v_{\varphi}-\frac{v_{\varphi}}{r^{2}}\right) \\
\frac{\partial v_{z}}{\partial t}=v \Delta v_{z}+g-\frac{1}{\rho} \frac{\partial P}{\partial z} \\
\frac{\partial P}{\partial r}=-\rho \frac{v_{\varphi}^{2}}{r} .
\end{array}\right.
$$

Поскольку мы рассматриваем ограниченную область размером $R_{1} \leq r \leq R_{2}$, то в (28) под $r$ следует понимать радиус-вектор, отсчитываемый в радиальном направлении от поверхности внутреннего цилиндра, т.е. $r^{\prime}=R_{1}+r$, где $r^{\prime}$ меняется в сегменте $R_{1} \leq r^{\prime} \leq R_{2}$, а $r$ - на радиальном сегменте $0 \leq r \leq R_{2}-R_{1}$. Везде далее, таким образом, под $R_{2}$ мы будем понимать разность $R_{2}-R_{1}$. Поскольку обе проекции скорости могут зависеть только от радиальной координаты, запишем систему (28) в виде

$$
\left\{\begin{array}{l}
\frac{\partial v_{\varphi}}{\partial t}=v\left(\frac{1}{r} \frac{\partial}{\partial r}\left(r \frac{\partial v_{\varphi}}{\partial r}\right)-\frac{v_{\varphi}}{r^{2}}\right) \\
\frac{\partial v_{z}}{\partial t}=\frac{v}{r} \frac{\partial}{\partial r}\left(r \frac{\partial v_{z}}{\partial r}\right)+g-\frac{1}{\rho} \frac{\partial P}{\partial z} \\
\frac{\partial P}{\partial r}=-\rho \frac{v_{\varphi}^{2}}{r}
\end{array}\right.
$$

Начнем с верхнего уравнения. Если искать решение стандартным методом разделения переменных [4], т.е. в виде $v_{\varphi}=u(r) p(t)$, немедленно получаем, что $p(t)=e^{-\lambda^{2} t}$, а для функции $u(r)$ находим уравнение

$$
r^{2} u^{\prime \prime}+r u^{\prime}+\left(\frac{\lambda^{2} r^{2}}{v}-1\right) u=0 .
$$

Это уравнение Бесселя и его решение можно представить как

$$
u=C_{1} J_{1}\left(\frac{r \lambda}{\sqrt{v}}\right)+C_{2} Y_{1}\left(\frac{r \lambda}{\sqrt{v}}\right)
$$

где $J_{1}(x)$ и $Y_{1}(x)$ - соответственно функции Бесселя первого и второго рода. В рамках решаемой нами задачи, когда граничные и начальные условия задаются в виде

$$
\begin{gathered}
\left.v_{\varphi}\right|_{r=R_{2}-R_{1}}=0, \\
\left.v_{\varphi}\right|_{r=0}=\varphi(t) R_{1}, \\
\omega(0)=\omega,
\end{gathered}
$$

где $\omega-$ постоянная частота вращения внутреннего цилиндра, имеется некоторый произвол с выбором констант $C_{1}$ и $C_{2}$. Это позволяет упростить решение и выбрать $C_{2}=0$. Поэтому решение в общем виде можно записать, как (ср. с [5])

$$
v_{\varphi}(r, t)=C_{1} e^{-\lambda^{2} t} J_{1}\left(\frac{r \lambda}{\sqrt{v}}\right) .
$$

Из условия (30) следует, что

$$
\lambda=\lambda_{k}=\mu_{k} \frac{\sqrt{\nu}}{R_{2}-R_{1}},
$$

где $\mu_{k}$ - корни уравнения $J_{1}(x)=0$, а индекс $k$ принадлежит полусегменту $k=[1, \infty)$. Поэтому решение (33) можно представить так

$$
v_{\varphi}(r, t)=\sum_{k=1}^{\infty} C_{k} e^{-\frac{\mu_{k}^{2} v t}{\left(R_{2}-R_{1}\right)^{2}}} J_{1}\left(\mu_{k} \frac{r}{R_{2}-R_{1}}\right) .
$$

Из второго граничного условия (31) получаем закон изменения частоты вращения слоев жидкости от времени, а именно

$$
\omega(t)=\frac{1}{R_{2}} \sum_{k=1}^{\infty} C_{k} e^{-\frac{\mu_{k}^{2} v t}{\left(R_{2}-R_{1}\right)^{2}}} J_{1}\left(\mu_{k} \frac{R_{1}}{R_{2}-R_{1}}\right) .
$$

Наконец, пользуясь начальным условием (32), имеем $C_{k}=a_{k} \omega R_{1}$, где коэффициенты $a_{k}$ удовлетворяют уравнению

$$
\sum_{k=1}^{\infty} a_{k} J_{1}\left(\mu_{k} \frac{R_{1}}{R_{2}-R_{1}}\right)=1 .
$$

Надо сказать, что уравнение (36), вообще говоря, имеет бесчисленное множество решений, но это вовсе не означает, что среди них нельзя выбрать какое-либо конкретное решение и остановиться на нем в качестве искомого. В самом деле, если, например, имеется ряд Римана

$$
\xi(p)=\sum_{n=1}^{\infty} \frac{1}{n^{p}},
$$

где $p>1$, то для вполне определенных значений параметра $p$ значение этой суммы известно. К примеру, если $p=2$, то $\xi(2)=\frac{\pi^{2}}{6}$. Возьмем самый простой вариант и выберем в качестве решения сумму вида

$$
a=\sum_{k=1}^{\infty} \frac{(-1)^{k}}{k}=\frac{\pi}{4} .
$$


Это означает, что уравнение (36) можно записать как

$$
\sum_{k=1}^{\infty} a_{k} J_{1}\left(\mu_{k} \frac{R_{1}}{R_{2}-R_{1}}\right)=\frac{4}{\pi} \sum_{k=1}^{\infty} \frac{(-1)^{k}}{k} .
$$

Откуда сразу следует, что

$$
a_{k}=\frac{4}{\pi} \frac{(-1)^{k}}{k J_{1}\left(\mu_{k} \frac{R_{1}}{R_{2}-R_{1}}\right)} .
$$

Следовательно, искомое решение задачи, согласно (34) и (35), можно представить в виде

$$
\begin{aligned}
v_{\varphi}(r, t)= & \frac{4 \pi R_{1}}{\pi} \sum_{k=1}^{\infty} \frac{(-1)^{k}}{k J_{1}\left(\mu_{k} \frac{R_{1}}{R_{2}-R_{1}}\right)} \\
& \times e^{-\frac{\mu_{k}^{2} v t}{\left(R_{2}-R_{1}\right)^{2}}} J_{1}\left(\mu_{k} \frac{r}{R_{2}-R_{1}}\right), \\
\omega(t)= & \frac{4 \omega R_{1}}{\pi\left(R_{2}-R_{1}\right)} \sum_{k=1}^{\infty} \frac{(-1)^{k}}{k J_{1}\left(\mu_{k} \frac{R_{1}}{R_{2}-R_{1}}\right)} \\
& \times e^{-\frac{\mu_{k}^{2} v t}{\left(R_{2}-R_{1}\right)^{2}}} J_{1}\left(\mu_{k} \frac{r}{R_{2}-R_{1}}\right) .
\end{aligned}
$$

Вернемся теперь снова к уравнениям (29). Поскольку изменение давления по глубине подчиняется уравнению $\frac{1}{\rho} \frac{\partial P}{\partial z}=g$, то второе уравнение в (29) дает нам

$$
\frac{\partial v_{z}}{\partial t}=\frac{v}{r} \frac{\partial}{\partial r}\left(r \frac{\partial v_{z}}{\partial r}\right)
$$

Так как нагревом жидкости мы пренебрегаем, то это уравнение выполняется автоматически, поскольку $v_{z}=0$, в силу того, что для вертикального перемещения жидкости нет никаких причин. Нижнее уравнение системы (29) позволяет выяснить распределение давления между цилиндрами. Действительно, поскольку

$$
\frac{d P}{d r}=-\rho \frac{v_{\varphi}^{2}}{r}
$$

то, подставив сюда решение (38), после интегрирования по $r$ получим

$$
\begin{aligned}
P(r, t)=P_{0} & -\frac{16 \pi \omega^{2} R_{1}^{2}}{\pi^{2}} \sum_{k, k^{\prime}}^{\infty}(-1)^{k+k^{\prime}} \\
& \times \frac{e^{-\frac{\left(\mu_{k}^{2}+\mu_{k^{\prime}}^{2}\right) v t}{\left(R_{2}-R_{1}\right)^{2}}} Q_{k k^{\prime}}(r)}{J_{1}\left(\mu_{k} \frac{R_{1}}{R_{2}-R_{1}}\right) J_{1}\left(\mu_{k^{\prime}} \frac{R_{1}}{R_{2}-R_{1}}\right)},
\end{aligned}
$$

где матрица

$$
Q_{k k^{\prime}}(r)=\int_{R_{1}}^{r} J_{1}\left(\mu_{k} \frac{R_{1}}{R_{2}-R_{1}}\right) J_{1}\left(\mu_{k^{\prime}} \frac{R_{1}}{R_{2}-R_{1}}\right) \frac{d r}{r} .
$$

Как видно из решений (38) и (39), время, через которое будет вовлечен во вращение внешний цилиндр, будет определяться самым максимальным временем $\tau_{k}$, которое определяется, как

$$
\tau_{k}=\frac{\left(R_{2}-R_{1}\right)^{2}}{\mu_{k}^{2} v} .
$$

Понятно, что максимальное время определится минимальным значением корня $\mu_{k}$, которое для уравнения $J_{1}(x)=0$ соответствует значению (см. [4]) $\mu_{1}=3.9$. Таким образом,

$$
\tau_{1}=\tau_{\max }=\frac{\left(R_{2}-R_{1}\right)^{2}}{3.9^{2} v} .
$$

Если для примера взять $R_{2}=10 \mathrm{~cm}, R_{1}=2 \mathrm{~cm}$, а кинематическую вязкость положить равной $v=1 \mathrm{~cm}^{2} / \mathrm{s}$, то получим $\tau_{1} \approx 4 \mathrm{~s}$. Этот результат можно легко проверить экспериментально. Фактически формулами (38)-(41) решение задачи может считаться законченным. Нам осталось только подвести некоторые итоги проведенного выше анализа и дать им краткую формулировку.

\section{Заключение}

1. При помощи предположения (7) найдено распределение гидродинамических скоростей в непосредственной близости от поверхности вращающегося цилиндра (ср. также с вычислениями, проведенными в [3]) и благодаря им вычислена диссипативная функция $\dot{Q}$. С ее помощью получено простое дифференциальное уравнение, описывающее движение вращающегося цилиндра в вязком континууме, и аналитически оценено время его остановки.

2. Найдено решение нестационарной задачи и оценен временной интервал, по прошествии которого начнет вовлекаться в движение внешний соосный с ним и неподвижный цилиндр. Отметим, что решение этой задачи позволяет легко наметить алгоритм решения и других подобных задач в этом направлении и, в частности, оценить время синхронизации маятников часов.

\section{Список литературы}

[1] Ландау Л.Д., Лифшии, Е.М. Гидродинамика. Т. 6. М.: Наука, 1988. $733 \mathrm{c}$.

[2] Мак Коннел А.Джс. Введение в тензорный анализ с приложениями к геометрии, механике и физике. М.: Физматлит, 1963. $411 \mathrm{c}$.

[3] Gladkov S.O. // Intern. J. Mathematical Models and Methods in Applied Sciences. 2015. Vol. 9. P. 166-170.

[4] Кошляков С.И., Глинер Э.Б., Смирнов М.М. Уравнения в частных производных математической физики. М.: Высшая шк., $1971.736 \mathrm{c}$.

[5] Гладков С.О. // Письма в ЖТФ. 2005. Т. 31. Вып. 12. С. $71-$ 75. 